\title{
Fractional Transportation Problem with Non-Linear Discount Cost
}

\author{
Ather Aziz Raina*, Srikant Gupta, Kirandeep Kour \\ *Department of Applied Mathematics, Govt. Post Graduate College \\ Rajouri, Jammu \& Kashmir (India) \\ Department of Statistics \& Operations Research, \\ Aligarh Muslim University, Aligarh (India) \\ Department of Statistics, \\ University of Jammu, Jammu \& Kashmir (India) \\ *Corresponding Author: ather.raina@yahoo.in \\ iD https://orcid.org/0000-0003-0034-8595
}

Received: $07^{\text {th }}$ September 2017/ Revised: $29^{\text {th }}$ of November 2018 / Published: $07^{\text {th }}$ of December 2018 CIAppStat-SL2017

\begin{abstract}
The generalization of linear programming is a fractional programming where the objective function is a proportion of two linear functions. Likewise, in fractional transportation problem the aim is to optimize or improve the ratio of two cost functions or damage functions or demand functions. Since the ratio of two functions is considered, the fractional programming models become more appropriate for dealing with real life problems. The fractional transportation problem (FTP) plays a very important role in supply management for reducing cost and amending service. In real life, the parameters in the models are rarely known exactly and have to be evaluated. This paper investigates the fractional transportation problem (FTP) with some discount cost that avails during the shipment time. The transportation problem, which is one of integer programming problems, deals with distributing any commodity from any group of 'sources' to any group of destinations or 'sinks' in the most effective way with a given 'supply' and 'demand' constraints. The volume of goods to be transported from one place to another incurs some discount cost that could effectively reduce the shipment cost which is directly related to the profit associated with the shipment. This paper is aimed at studying the optimal solution for the problem has been achieved by using Karush-Kuhn-Tucker (KKT) optimality algorithm. Finally, a numerical example is illustrated to support the algorithm.
\end{abstract}

Keywords: Fractional Programming, Transportation Problem, Non-linear Programming, Karush-Kuhn-Tucker (KKT). 


\section{Introduction}

Fractional Programming Problem (FPP) is a mathematical language used for describing the optimization problems. It is based on parameters, decision variables, objective function (rational form) subject to various types of constraints. FPP is an extension or generalization of linear programming problem where the objective function is in the form of ratio with linear constraints. The fractional programming problem has received the interest of many researchers due to its application in numerous essential fields such as production planning, financial and corporate planning, health care and hospital planning. It can be seen in many practical optimization problems where the objective functions are quotients of two functions which are used to achieve the highest ratio of outcome to cost, profit to time, profit to cost, output to employees, return to cost, actual cost to standard cost and minimizing inventory to sales, cost to time, student to teacher ratios etc., where the ratio representing the highest efficiency of a system. Several methods were suggested for solving this problem such as the variable transformation method Charnes and Cooper (1962) and the updated objective function method by Bitran and Novaes (1973).

Transportation problem is one of the most widely used applications of mathematical programming. Mainly, transportation problem is concerned with shipping methods or selecting routes in a product distribution network among the manufacturing plants and distribution warehouses situated in different regions or local outlets. In literature, it has been used in number of ways. The problem commonly faced in the distribution of goods from various supply points to different destination points is known as the transportation problem, first studied by Hitchcock (1941). Dantzig (1951) adapted the simplex method to solve the transportation problem formulated earlier by Hitchcock. Charnes et al. (1954) derived an intuitive presentation of Dantzig's procedure called the stepping-stone method which follows the basic logic of the simplex method but avoids the use of the tableau and the pivot operations required to get the inverse of the basis.

Originally, Swarup (1966) studied a fractional transportation problem under the assumption that the denominator is always positive and it has an important role in logistics and supply chain management for reducing cost and improving service. Transportation problem with discount cost has been studied by many researchers viz., Shetty (1959) formulated an algorithm to solve transportation problems taking non-linear costs. $\mathrm{He}$ considered the case when a convex production cost is included at each supply centre besides the linear transportation cost. Dhingra, et al.(2012) have proposed an algorithm to solve multi-objective fractional bottleneck transportation problem with restrictions. Haruna et al. (2012) gave an algorithm for a transportation problem with non-linear discount cost 
and applied this algorithm successfully on GALCO Company Limited (Kumasi, Ghana).Jain et al. (2012) have studied the time minimizing transportation problem with fractional bottleneck objective function. Nuran et al. (2012) have obtained a solution proposal to the interval fractional transportation problem. Acharya et al. (2013) developed a new algorithm for obtaining the optimum solution of discounted generalized transportation problem. Ekezie et al. (2013) focused on the application of transportation problem with volume discount on distribution cost using Porthar Court Flour Mills Company.

Mubashiru (2014) focused on the model of the non-linear transportation problem for a particular company in Ghana, where he used KKT optimality algorithm to solve the company's problem. Singh (2015) have studied the multiple-objective fractional costs transportation problem with bottleneck time and impurities. Saifet al. (2017) have discussed the uncertainty in linear fractional transportation problem. Sadia et al. (2018) have considered a multi-objective fractional transportation problem in fuzzy environment. Liu (2016) have presented a fractional transportation problem with fuzzy parameters. A non-linear version of the transportation problem with a fixed number of sources was studied by Cosares et al. in (1995). Other approaches to the non-linear variants of the transportation problem were discussed by Ilich et al. (2001), Jo et al. (2007), Klansek et al.(2010)and Anholcer (2015).

In this paper, we have proposed a method for fractional transportation problem to find out the per unit profit associated with each shipping along with some non-linear discount cost. We have also used KKT condition to get the optimal solution. The methodology has been verified through a numerical example.

This paper is organized in the following way. In Section 1, we have presented a background study of fractional programming problem and transportation problem. In Section 2, preliminaries used for solving fractional transportation problems and the KKT optimality condition have been discussed. In Section 3, algorithms for solving the fractional transportation problems are presented. The effectiveness of the algorithm is proven by the practical application in section 4 . Finally, in section 5, concluding remarks are outlined.

\section{Preliminaries}

This research seeks to apply the existing general non-linear programming algorithms to solve the problem. Before formulating the problem of interest, it is necessary to have a 
good understanding of the background of non-linear programming which is reproduced here from Mubashiru (2014):

\subsection{Polyhedral Sets}

A set $P$ in an $n$ dimensional normed vector space $E^{n}$ is called polyhedral set if it is the intersection of a finite number of closed-half spaces, i.e. $P=\left\{X: P_{i} X \leq a_{i}, i=1,2, \ldots, m\right\}$ where, $P_{i}$ is a non-zero vector in $E^{n}$ and $a_{i}$ is a scalar. A polyhedral set is a closed convex set and can be represented by a finite number of inequalities and/or equations.

We consider the polyhedral set $\{x: A x=b, x \geq 0\}$; where, $A$ is an $m \times n$ matrix and $b$ is an $m$-vector, also assume that the rank of $A$ is $m$. If not, assuming that $A x=b$ is consistent, we can leave aside any redundant equations. Let $\mathrm{P}$ be non empty convex set in $E^{n}$. A vector $x \in P$ is called an extreme point of $P$ if $x=\beta x_{1}+(1-\beta) x_{2}$ with $x_{1}$ and $x_{2}$ elements of $P$ and $\beta \in(0,1)$.

Theorem 1: Let $P=\{x: A x=b, x \geq 0\}$ be a non-empty polyhedral set in $E^{n}$ where, $A$ ism $\mathrm{x} n$ matrix of rank $m$, and $b$ is an $m$ vector. A point $x$ is an extreme point of $P$ if and only if $A$ can be decomposed into $[B, N]$ such that,

$$
x=\left(\begin{array}{l}
x B \\
x N
\end{array}\right)=\left(\begin{array}{l}
B-1 b \\
0
\end{array}\right)
$$

where, $B$ is an $m \times n$ invertible matrix satisfying $B^{-1} b \geq 0$. Any such solution is called a basic feasible solution for $P$. The number of extreme points of $P$ is finite.

Theorem 2 (Existence of extreme points): Let $P=\{x: A x=b, x \geq 0\}$ be a nonemptypolyhedral setin $E^{n}$ where, $A$ is an $m \times n$ matrix of rank $m$ and $b$ is an $m$ vector. Then $P$ has at least one extreme point.

\subsection{Extreme Direction}

Let $P$ be a non-empty polyhedral set in $E^{n}$. A non-zero vector $d$ in $E^{n}$ is called direction or recession direction of $P$ if $x+\beta d \in P$ for each $x \in P$ and for all $\beta \geq 0$. Therefore, it follows that, $d$ is a direction of $P$ if and only if $A d=0$ and $d \geq 0$. 
Theorem 3(Characterization of Extreme Directions): Let $P=\{x: A x=b, x \geq 0\}$ be $a$ non-empty polyhedral set in $E^{n}$ where, $A$ is an $m \mathrm{x}$

$n$ matrix of rank $m$ and $b$ is an $m$ vector. A vector $\bar{d}$ is an extreme direction of $P$ if and only if $A$ can be decomposed into $[B, N]$ such that $B^{-1} a_{j} \leq 0$ for some column $a_{j}$ of $N$ and $\bar{d}$ is a positive multiple of $d=\left(\begin{array}{c}B-1 a_{j} \\ e_{j}\end{array}\right)$ where, $e_{j}$ is an $n$ - $m$ vector of zero except for in position $j$ which is 1 .

Theorem 4(Representation theorem):Let $P=\{x: A x=b, x \geq 0\}$ be a nonemptypolyhedral set in $E^{n}$ and let $x_{1}, x_{2}, \ldots, x_{k}$ be the extreme points of $P$ and $d_{1}, d_{2}, \ldots, d_{l}$ be the extreme direction of $P$. Then $x \in P$ if and only if $x$ can be written as:

$$
\begin{aligned}
& x=\sum_{j=1}^{k} \beta_{j} x_{j}+\sum_{i=1}^{l} \gamma_{i} d_{i} \\
& \sum_{j=1}^{k} \beta_{j}=1, \beta_{j} \geq 0, \quad \gamma_{i} \geq 0
\end{aligned}
$$

Theorem 5(Existence of Extreme directions): Let $P=\{x: A x=b, x \geq 0\}$ be a nonempty polyhedral set in $E^{n}$ where, $A$ is an $m \times n$ matrix with rank $m$. Then, $P$ has at least one extreme direction if and only if it is unbounded.

\subsection{The Karush-Kuhn-Tucker (KKT) Optimality Condition for Non-Linear Programming Problem (NPP)}

Given the non-linear programming problem:

$$
\begin{array}{lll}
(N P P) & \min f(x) & \\
\text { s.t. } & g_{i}(x) \leq 0, & i=1,2, \ldots, k \\
& h_{j}(x)=0, & j=1,2, \ldots, l
\end{array}
$$

In the following subsections, we have discussed about the necessary and sufficient optimality conditions of KKT. 


\subsubsection{KKT Necessary Optimality Conditions}

Theorem 5: Given the objective function $f: \mathbb{R}^{n} \rightarrow \mathbb{R}$ and the constraint functions are $g_{i}: \mathbb{R}^{n}$ $\rightarrow \mathbb{R}$ and $h_{j}: \mathbb{R}^{n} \rightarrow \mathbb{R}$ and $I=\left\{i: g_{i}\left(x^{*}\right)=0\right\}$. In addition, suppose they are continuously differentiable at a feasible point $x^{*}$ and $\nabla g_{i}\left(x^{*}\right)$ for $i \in I$ and $\nabla h_{j}\left(x^{*}\right)$ for $j=1,2, \ldots$, l be linearly independent. If $x^{*}$ is minimize of the problem (NPP), then there exist scalars $\bar{\lambda}_{i}, \quad i=1,2, \ldots, k$ and $\bar{\mu}_{j}, \quad j=1,2, \ldots$, l minimize called Lagrange multipliers, such that,

$$
\begin{gathered}
\Delta f\left(x^{*}\right)+\sum_{i=1}^{k} \bar{\lambda}_{i} \nabla g_{i}\left(x^{*}\right)+\sum_{j=1}^{l} \bar{\mu}_{j} \nabla h_{j} x^{*}=0 \\
\bar{\lambda}_{i} g_{i}\left(x^{*}\right)=0, \bar{\lambda}_{i} \geq 0 ; \bar{\mu}_{j} \in \mathbb{R}
\end{gathered}
$$

Further, iff and $g_{i}$ are convex, each $h_{j}$ as affine, then the above necessary optimality condition will also be sufficient.

\subsubsection{KKT Sufficient Optimality Conditions}

Let $x$ be any feasible point different form $x *$. From the first KKT conditions, we obtain,

$$
\nabla f\left(x^{*}\right)\left(x-x^{*}\right)=-\left(\sum_{i=1}^{k} \bar{\lambda}_{i} \nabla g_{i}\left(x^{*}\right)+\sum_{j=1}^{l} \bar{\mu}_{j} \nabla h_{j}\left(x^{*}\right)\left(x-x^{*}\right)\right)
$$

Since, each $g_{i}(x)$ is convex, $\bar{\lambda}_{i} \geq 0$, we have, $\nabla h_{j}\left(x^{*}\right)\left(x-x^{*}\right)=0 \forall j$.

Also,

$$
\sum_{i=}^{k} \bar{\lambda}_{i} \nabla g_{i}\left(x^{*}\right)\left(x-x^{*}\right) \leq \sum_{i=1}^{k} \bar{\lambda}_{i}\left[g_{i}(x)-g_{i}\left(x^{*}\right)\right] \Rightarrow \nabla f\left(x^{*}\right)\left(x-x^{*}\right) \geq-\sum \bar{\lambda}_{i} g_{i}(x) \geq 0
$$

rom convexity of $f(x)$, we get,

$$
f(x)-f\left(x^{*}\right) \geq 0 \Leftrightarrow f\left(x^{*}\right) \leq f(x) \text { for any feasible } x .
$$




\subsubsection{KKT Optimality Condition for Fractional Transportation Problem}

The general fractional transportation problem is modeled as:

$$
\begin{aligned}
& \operatorname{Max}(\text { or Min }) Z=\frac{P(x)}{C(x)}=\frac{\sum_{i=1}^{m} \sum_{j=1}^{n} p_{i j} x_{i j}+p_{0}}{\sum_{i=1}^{m} \sum_{j=1}^{n} c_{i j} x_{i j}+c_{0}} \\
& \text { s.t. } \quad \sum_{j=1}^{n} x_{i j} \leq a_{i}, i=1,2, \ldots, m \\
& \sum_{i=1}^{m} x_{i j} \geq b_{j}, j=1,2, \ldots, n \\
& x_{i j} \geq 0, i=1,2, \ldots, m ; j=1,2, \ldots, n
\end{aligned}
$$

We suppose that $D(x)>0, \forall x=\left(x_{i j}\right) \in S$, where, $S$ denotes a feasible set defined by constraints. Further, we assume that $a_{i}>0, b_{j}>0, i=1,2, \ldots, m ; j=1,2, \ldots, n$ and total supply is not less than total demand, i.e.,

$$
\sum_{i=1}^{m} a_{i} \geq \sum_{j=1}^{n} b_{j}
$$

The fractional transportation table can be represented as:

\begin{tabular}{|c|c|c|c|c|c|}
\hline$\frac{\partial Z(\bar{x})}{\partial x_{11}}$ & $\ldots$ & $\ldots$ & $\frac{\partial Z(\bar{x})}{\partial x_{1 m}}$ & $a_{1}$ & $u_{1}$ \\
\hline$\ldots$ & $\ldots$ & $\ldots$ & $\ldots$ & $\ldots$ & $\ldots$ \\
\hline$\ldots$ & $\frac{\partial Z(\bar{x})}{\partial x_{i j}}$ & $\ldots$ & $\ldots$ & $a_{i}$ & $u_{i}$ \\
\hline$\frac{\partial Z(\bar{x})}{\partial x_{n 1}}$ & $\ldots$ & $\ldots$ & $\frac{\partial Z(\bar{x})}{\partial x_{n m}}$ & $a_{n}$ & $u_{n}$ \\
\hline$b_{1}$ & $\ldots$ & $\ldots$ & $b_{m}$ & & \\
\hline$v_{1}$ & $\ldots$ & $\ldots$ & $v_{m}$ & & \\
\hline
\end{tabular}

where, $\bar{x}$ is the current basic solution. 
The Lagrange function for the non-linear fractional transportation is formulated in the form:

$$
Z(x, \lambda, w)=\frac{p(x)}{c(x)}+w(b-A x)-\lambda x
$$

where, $\lambda$ and $w$ are Lagrange multipliers and $\lambda \in \mathbb{R}^{\mathrm{nm}}$.

The optimal point $\bar{x}$ should satisfy the KKT conditions:

$$
\begin{gathered}
\nabla Z=\nabla \frac{p(\bar{x})}{c(\bar{x})}-\mathrm{w}^{\mathrm{T}} \mathrm{A}-\lambda=0 \\
\lambda \bar{x}=0, \lambda \geq 0, \bar{x} \geq 0 .
\end{gathered}
$$

Specifically, for each cell $(i, j)$, we have,

$$
\begin{gathered}
\frac{\partial Z}{\partial x_{i j}}=\frac{\partial Z(\bar{x})}{\partial x_{i j}}-(u, v)\left(e_{i}, e_{n+j}\right)-\lambda_{i j}=0 \\
\lambda_{i j} x_{i j}=0, x_{i j} \geq 0, \lambda_{i j} \geq 0 .
\end{gathered}
$$

where, $k=1, \ldots, n m$ and $w=(u, v)=\left(u_{1}, u_{2}, \ldots, u_{n}, v_{1}, v_{2}, \ldots, v_{m}\right), e_{k} \in \mathbb{R}^{\mathrm{m}+\mathrm{n}}$ is a vector of zeros except at position $k$ which is 1 . From the condition (3) and $\lambda \geq 0$, we get,

$$
\begin{gathered}
\frac{\partial Z}{\partial x_{i j}}=\frac{\partial Z(\bar{x})}{\partial x_{i j}}-u_{i}-v_{j} \geq 0, \\
x_{i j} \frac{\partial Z}{\partial x_{i j}}=x_{i j} \frac{\partial Z(\bar{x})}{\partial x_{i j}}-u_{i}-v_{j}=0, x_{i j} \geq 0 .
\end{gathered}
$$

\section{General solution procedure for the NTP:}

- Initialization: Find an initial basic feasible solution $\bar{x}$.

- Iteration: Step 1- If $\bar{x}$ is KKT point, stop. Otherwise, go to the next step.

Step 2- Find the new feasible solution that improves the cost function and go to step 1.

\section{Algorithm for Solving Non-linear Fractional Transportation Problem}

Step (1): First convert the problem into $\left(\frac{p_{i j}}{c_{i j}}\right)$ form. 
Step (2): Find the initial basic feasible solution of the FTP by using anyone of the wellknown method for solving transportation problem.

Step (3): Determine the value of objective function at $\bar{x}$ i.e. $Z=\frac{p(\bar{x})}{c(\bar{x})}$

\section{Test for Optimality (or improvement):}

After obtaining the initial feasible solution, the next step is to test whether it is optimal or not. If the solution is non-optimal, then we improve the solution by exchanging non-basic variable for a basic variable. In other words, we rearrange the allocation by transferring units from an occupied cell to an empty cell that has the largest net cost change or improvement index and then shift the units from other related cells so that all the rim (supply, demand) requirements are satisfied. This is done by tracing a closed path or closed loop.

Step (4): Add a $u_{i}$ to the RHS of the transportation tableau and an $v_{j}$ at the bottom of the tableau.

Step (5): Determine the values of $u_{i}$ and $v_{j}$ by using the relationship,

$$
\frac{\partial f}{\partial x_{B_{i j}}}=u_{i}+v_{j}
$$

We can determine the values of $u_{i}$ and $v_{j}$ by assigning one $u_{i}$ 's the zero value. Also, $\frac{\partial f}{\partial x_{B_{i j}}}$ values can be obtained from non-linearity condition defined earlier, where $x_{B_{i j}}$ are the basic variables.

Step (6): If $Q_{i j}=\frac{\partial Z}{\partial x_{N B_{i j}}}-u_{i}-v_{j} \geq 0$ for all $x_{i j}$ non-basic, $\bar{x}$ is KKT point.

Otherwise, go to step (7), where, $x_{N B_{i j}}$ are the non-basic variables.

Step (7): Check the following given conditions:

1. If all $\frac{\partial f}{\partial x_{N B_{i j}}}>0$, the solution under test is optimal and unique.

2. If all $\frac{\partial f}{\partial x_{N B_{i j}}} \geq 0$, with at least one $\frac{\partial f}{\partial x_{N B_{i j}}}=0$, the solution under test is optimal and alternative solution exist. 
3. If at least one $\frac{\partial f}{\partial x_{N B_{i j}}}<0$, the solution is not optimal.

Calculate $\frac{\partial Z}{\partial x_{r l}}=\min \left\{\frac{\partial Z(\bar{x})}{\partial x_{i j}}-\left(u_{i}+v_{j}\right)\right\} x_{r l}$ will enter the basis. Allocate $x_{r l}=\theta$, where, $\theta$ is found as in the linear transportation case. Adjust the allocation so that the constraints are satisfied. Determine the leaving variable say, $x_{B_{r k}}$, where, $x_{B_{r k}}$ is the basic variable comes to zero first while making the adjustment. Then, find the new basic variable and go to step 1 .

The feasible set of our problem is a non-empty polyhedral set. And by definition, a polyhedral set $P$ is a set bounded with a finite number of hyper planes from which it follows that it possesses finite number of extreme points. In each step of the algorithm, we jump from one extreme point to another looking for a better feasible solution implying that the algorithm will terminate after a finite iteration. In addition, since for all $i$ and $j$, $0 \leq x_{i j} \leq \max \left\{a_{i}, b_{j}\right\}, P$ is bounded that guarantees the existence of minimum value.

\section{Numerical Example}

In this section, we consider a computational study of the above solution procedures. Emphasis will be given to a FTP where discounts are given to volume on quantity of goods transported which is concave in nature. Consider the following FTP with profit and shipping cost. We formulate the problem to maximize the per unit profit associated with shipments. Profit matrix $P=\left\|p_{i j}\right\|_{m \times n}$ which determines the profit $p_{i j}$ gained by a transportation company if a unit of the good is shipped from supply point $i$ to demand point $j$ are shown in Table 1 .

Table 1: Profit associated with shipments, where A, B, C and D are sinks and E, F and $G$ are sources with cost (in Rs.) of shipments

\begin{tabular}{|c|c|c|c|c|c|}
\hline & A & B & C & D & Supply \\
\hline E & 12 & 18 & 10 & 14 & 150 \\
\hline F & 10 & 14 & 16 & 10 & 250 \\
\hline G & 11 & 8 & 17 & 11 & 200 \\
\hline Demand & 100 & 250 & 100 & 150 & 600 \\
\hline
\end{tabular}


Cost matrix $C=\left\|c_{i j}\right\|_{m \times n}$ which determines the cost $c_{i j}$ of shipping a unit of the good from supply point $i$ to demand point $j$ are shown in Table 2 .

Table 2: Cost of shipments (in Rs.) where A, B, C and D are sinks and E, F and $G$ are sources

\begin{tabular}{|c|c|c|c|c|c|}
\hline & A & B & C & D & Supply \\
\hline E & 17 & 14 & 18 & 10 & 150 \\
\hline F & 12 & 8 & 15 & 14 & 250 \\
\hline G & 15 & 17 & 14 & 12 & 200 \\
\hline Demand & 100 & 250 & 100 & 150 & 600 \\
\hline
\end{tabular}

Due to some shipping policy, there is a discount cost $D=\left\|d_{i j}\right\|_{m \times n}$ associated with each shipment and it is directly related to the unit of commodity purchased and transported and the discount (\%) are shown in Table 3.

Table 3: Discount Cost (\%) where A, B, C and D are sinks and E, F and $G$ are sources

\begin{tabular}{|c|c|c|c|c|}
\hline & $\mathbf{A}$ & $\mathbf{B}$ & $\mathbf{C}$ & $\mathbf{D}$ \\
\hline $\mathbf{E}$ & 0.02 & 0.03 & 0.05 & 0.02 \\
\hline $\mathbf{F}$ & 0.03 & 0.01 & 0.005 & 0.02 \\
\hline $\mathbf{G}$ & 0.014 & 0.04 & 0.013 & 0.04 \\
\hline
\end{tabular}

If we suppose that discount cost associated with each shipping from $i$ to $j$, then the nonlinear fractional transportation problem can be formulated as follows:

$$
\operatorname{Max} Z=\frac{P(x)}{C(x)}=\frac{\begin{array}{l}
12 x_{11}+18 x_{12}+10 x_{13}+14 x_{14}+10 x_{21}+14 x_{22}+11 x_{31}+8 x_{32}+17 x_{33}+11 x_{34} \\
17 x_{11}+14 x_{12}+18 x_{13}+10 x_{14}+12 x_{21}+8 x_{22}+ \\
15 x_{23}+14 x_{24}+15 x_{31}+17 x_{32}+14 x_{33}+12 x_{34}
\end{array}}{}
$$

subject to constraint 


$$
\begin{aligned}
& x_{11}+x_{12}+x_{13}+x_{14}=150, \\
& x_{21}+x_{22}+x_{23}+x_{24}=250, \\
& x_{31}+x_{32}+x_{33}+x_{34}=200, \\
& x_{11}+x_{21}+x_{31}=100, \\
& x_{12}+x_{22}+x_{32}=250, \\
& x_{13}+x_{23}+x_{33}=100, \\
& x_{14}+x_{24}+x_{34}=150 . \\
& x_{i j} \geq 0, i=1,2,3 ; j=1,2,3,4 .
\end{aligned}
$$

Therefore, we get the following terms of cost function:

$$
\begin{array}{ll}
\frac{p_{11}}{d_{11}} x_{11}=0.71 x_{11}-d_{11} x_{11}^{2}, & \frac{p_{23}}{d_{23}} x_{23}=1.1 x_{23}-d_{23} x_{23}^{2}, \\
\frac{p_{12}}{d_{12}} x_{12}=1.28 x_{12}-d_{12} x_{12}^{2}, & \frac{p_{24}}{d_{24}} x_{24}=0.71 x_{24}-d_{24} x_{24}^{2}, \\
\frac{p_{13}}{d_{13}} x_{13}=0.55 x_{13}-d_{13} x_{13}^{2}, & \frac{p_{31}}{d_{31}} x_{31}=0.73 x_{31}-d_{31} x_{31}^{2}, \\
\frac{p_{14}}{d_{14}} x_{14}=1.4 x_{14}-d_{14} x_{14}^{2}, & \frac{p_{32}}{d_{32}} x_{32}=0.47 x_{32}-d_{32} x_{32}^{2}, \\
\frac{p_{21}}{d_{21}} x_{21}=0.83 x_{21}-d_{21} x_{21}^{2}, & \frac{p_{33}}{d_{33}} x_{33}=1.22 x_{33}-d_{33} x_{33}^{2}, \\
\frac{p_{22}}{d_{22}} x_{22}=1.75 x_{22}-d_{22} x_{22}^{2}, & \frac{p_{34}}{d_{34}} x_{34}=0.92 x_{34}-d_{34} x_{34}^{2} .
\end{array}
$$

If we allow the discount on each transported product $i$ from the source to each of the destination $j$ as given in Table 3, the cost function becomes:

$$
\begin{array}{ll}
\frac{p_{11}}{d_{11}} x_{11}=0.71 x_{11}-0.02 x_{11}^{2}, & \frac{p_{23}}{d_{23}} x_{23}=1.1 x_{23}-0.005 x_{23}^{2}, \\
\frac{p_{12}}{d_{12}} x_{12}=1.28 x_{12}-0.03 x_{12}^{2}, & \frac{p_{24}}{d_{24}} x_{24}=0.71 x_{24}-0.02 x_{24}^{2},
\end{array}
$$




$$
\begin{array}{ll}
\frac{p_{13}}{d_{13}} x_{13}=0.55 x_{13}-0.05 x_{13}^{2}, & \frac{p_{31}}{d_{31}} x_{31}=0.73 x_{31}-0.014 x_{31}^{2}, \\
\frac{p_{14}}{d_{14}} x_{14}=1.4 x_{14}-0.02 x_{14}^{2}, & \frac{p_{32}}{d_{32}} x_{32}=0.47 x_{32}-0.04 x_{32}^{2}, \\
\frac{p_{21}}{d_{21}} x_{21}=0.83 x_{21}-0.03 x_{21}^{2}, & \frac{p_{33}}{d_{33}} x_{33}=1.22 x_{33}-0.013 x_{33}^{2}, \\
\frac{p_{22}}{d_{22}} x_{22}=1.75 x_{22}-0.01 x_{22}^{2}, & \frac{p_{34}}{d_{34}} x_{34}=0.92 x_{34}-0.04 x_{34}^{2} .
\end{array}
$$

\begin{tabular}{|c|c|c|c|c|c|c|c|c|c|}
\hline & \multicolumn{2}{|c|}{ A } & & 3 & \multicolumn{2}{|r|}{ C } & \multicolumn{2}{|r|}{ D } & Supply \\
\hline \multirow{2}{*}{ D } & 12 & 50 & \multirow{2}{*}{\multicolumn{2}{|c|}{14}} & 10 & 100 & \multirow{2}{*}{\multicolumn{2}{|c|}{14}} & \\
\hline & \multicolumn{2}{|c|}{17} & & & & 18 & & & 150 \\
\hline \multirow{2}{*}{$\mathbf{E}$} & 10 & 50 & \multicolumn{2}{|r|}{50} & & 16 & 10 & 150 & \multirow{2}{*}{250} \\
\hline & & 2 & & 8 & & 15 & & 14 & \\
\hline \multirow{2}{*}{$\mathbf{F}$} & & 1 & 8 & 200 & & 17 & & 11 & \multirow[b]{2}{*}{200} \\
\hline & & 5 & & 17 & & 14 & & 12 & \\
\hline Demand & & 00 & & 50 & & 100 & & 50 & 600 \\
\hline
\end{tabular}

Using any known method for solving transportation problem, we get the initial basic solution. The solution tableau is shown below:

Table 4: Solution Tableau

From the Table 4, the initial basic feasible solution is:

$$
\bar{x}=(50,0,100,0.50,50,0,150,0,200,0,0)
$$

And the value of objective function at $\bar{x}$ is:

$$
Z=\frac{P(x)}{C(x)}=\frac{12 \times 50+10 \times 100+10 \times 50+14 \times 50+10 \times 150+8 \times 200}{17 \times 50+18 \times 100+12 \times 50+8 \times 50+14 \times 150+17 \times 200}=0.6448 .
$$


Now, we use the KKT optimality conditions to improve our solution. The partial derivatives at $\bar{x}$ for the required function are given as:

$$
\begin{aligned}
& \frac{\partial Z}{\partial x_{11}}=-1.29, \frac{\partial Z}{\partial x_{12}}=1.28, \frac{\partial Z}{\partial x_{13}}=-9.45, \frac{\partial Z}{\partial x_{14}}=1.4, \frac{\partial Z}{\partial x_{21}}=-2.17, \frac{\partial Z}{\partial x_{22}}=0.75, \\
& \frac{\partial Z}{\partial x_{23}}=1.1, \frac{\partial Z}{\partial x_{24}}=-5.29, \frac{\partial Z}{\partial x_{31}}=0.73, \frac{\partial Z}{\partial x_{32}}=-15.53, \frac{\partial Z}{\partial x_{33}}=1.22, \frac{\partial Z}{\partial x_{34}}=0.92 .
\end{aligned}
$$

Next, we find the cost equation of the occupied cell using the equation

$$
\frac{\partial Z}{\partial x_{B_{i j}}}=u_{i}+v_{j}
$$

Using the above values of partial derivatives, we get,

$$
\begin{array}{ll}
\frac{\partial Z}{\partial x_{11}}=u_{1}+v_{1} \Rightarrow u_{1}+v_{1}=-1.29, & \frac{\partial Z}{\partial x_{13}}=u_{1}+v_{3} \Rightarrow u_{1}+v_{3}=-9.45 \\
\frac{\partial Z}{\partial x_{21}}=u_{2}+v_{1} \Rightarrow u_{2}+v_{1}=-2.17, & \frac{\partial Z}{\partial x_{22}}=u_{2}+v_{2} \Rightarrow u_{2}+v_{2}=0.75 \\
\frac{\partial Z}{\partial x_{24}}=u_{2}+v_{4} \Rightarrow u_{2}+v_{4}=-5.29, & \frac{\partial Z}{\partial x_{32}}=u_{3}+v_{2} \Rightarrow u_{3}+v_{2}=-15.53 .
\end{array}
$$

Letting $u_{1}=0$ in the above equations, we obtain the following values:

$$
\begin{gathered}
u_{1}=0, u_{2}=-0.88, u_{3}=-17.16, \\
v_{1}=-1.29, v_{2}=1.63, v_{3}=-9.45, v_{4}=-4.41
\end{gathered}
$$

Next, we find the net evaluation factor or reduced cost for the non-basic variables using the following equation:

$$
Q_{i j}=\frac{\partial Z}{\partial x_{N B_{i j}}}-u_{i}-v_{j}
$$


Therefore, after substituting the values of $u_{1}, u_{2}, u_{3}$ and $v_{1}, v_{2}, v_{3}, v_{4}$ in the above equation, we get,

$$
\begin{array}{cl}
Q_{12}=\frac{\partial Z}{\partial x_{12}}-u_{1}-v_{2}=-0.35, & Q_{14}=\frac{\partial Z}{\partial x_{14}}-u_{1}-v_{4}=5.81, \\
Q_{23}=\frac{\partial Z}{\partial x_{23}}-u_{2}-v_{3}=11.43, & Q_{31}=\frac{\partial Z}{\partial x_{31}}-u_{3}-v_{1}=19.18, \\
Q_{33}=\frac{\partial Z}{\partial x_{33}}-u_{3}-v_{3}=28.33, & Q_{34}=\frac{\partial Z}{\partial x_{34}}-u_{3}-v_{4}=22.99 .
\end{array}
$$

The presence of negative value for the reduced cost signifies non-optimality. From the above equations, the minimum reduced cost for the non-basic variable is $x_{12}$. Therefore, $x_{12}$ should enter the basis since it is the only negative reduced cost. We then move to the next iteration, the initial basic feasible solution is $\bar{x}^{1}=(0,50,100,0,100,0,0,100,0,200,0,0)$ and the value of objective function at $\bar{x}^{1}$ is:

$$
Z=\frac{P(x)}{C(x)}=\frac{8 \times 50+10 \times 100+10 \times 100+10 \times 100+8 \times 200}{14 \times 50+18 \times 100+12 \times 100+14 \times 100+17 \times 200}=0.5882
$$

Again, we use the KKT optimality conditions to improve our solution. The partial derivatives at $\bar{x}$ for the required function are given as:

$$
\begin{aligned}
& \frac{\partial Z}{\partial x_{11}}=0.71, \frac{\partial Z}{\partial x_{12}}=-1.72, \frac{\partial Z}{\partial x_{13}}=-9.45, \frac{\partial Z}{\partial x_{14}}=1.4, \frac{\partial Z}{\partial x_{21}}=-5.17, \frac{\partial Z}{\partial x_{22}}=1.75, \\
& \frac{\partial Z}{\partial x_{23}}=1.1, \frac{\partial Z}{\partial x_{24}}=-5.29, \frac{\partial Z}{\partial x_{31}}=0.73, \frac{\partial Z}{\partial x_{32}}=-15.53, \frac{\partial Z}{\partial x_{33}}=1.22, \frac{\partial Z}{\partial x_{34}}=0.92 .
\end{aligned}
$$

Next, we find the cost equation of the occupied cell using the following equation:

$$
\frac{\partial Z}{\partial x_{B_{i j}}}=u_{i}+v_{j}
$$


Using the above values of partial derivatives, we get,

$$
\begin{array}{ll}
\frac{\partial Z}{\partial x_{12}}=u_{1}+v_{2} \Rightarrow u_{1}+v_{2}=-1.72, & \frac{\partial Z}{\partial x_{13}}=u_{1}+v_{3} \Rightarrow u_{1}+v_{3}=-9.45, \\
\frac{\partial Z}{\partial x_{21}}=u_{2}+v_{1} \Rightarrow u_{2}+v_{1}=-5.17, & \frac{\partial Z}{\partial x_{22}}=u_{2}+v_{2} \Rightarrow u_{2}+v_{2}=1.75, \\
\frac{\partial Z}{\partial x_{24}}=u_{2}+v_{4} \Rightarrow u_{2}+v_{4}=-5.29, & \frac{\partial Z}{\partial x_{32}}=u_{3}+v_{2} \Rightarrow u_{3}+v_{2}=-15.53 .
\end{array}
$$

Letting $u_{1}=0$ in the above equations, we obtain the following values:

$$
\begin{gathered}
u_{1}=0, u_{2}=3.47, u_{3}=-13.81 \\
v_{1}=-8.64, v_{2}=-1.72, v_{3}=-9.45, v_{4}=-8.75 .
\end{gathered}
$$

Now, we find the net evaluation factor or reduced cost for the non-basic variables using the following equation:

$$
Q_{i j}=\frac{\partial Z}{\partial x_{N B_{i j}}}-u_{i}-v_{j}
$$

Substituting the values of $u_{1}, u_{2}, u_{3}$ and $v_{1}, v_{2}, v_{3}, v_{4}$ in the above equation, we get,

$$
\begin{array}{ll}
Q_{11}=\frac{\partial Z}{\partial x_{11}}-u_{1}-v_{1}=9.35, & Q_{14}=\frac{\partial Z}{\partial x_{14}}-u_{1}-v_{4}=10.15 \\
Q_{23}=\frac{\partial Z}{\partial x_{23}}-u_{2}-v_{3}=7.08, & Q_{31}=\frac{\partial Z}{\partial x_{31}}-u_{3}-v_{1}=23.18 \\
Q_{33}=\frac{\partial Z}{\partial x_{33}}-u_{3}-v_{3}=24.48, & Q_{34}=\frac{\partial Z}{\partial x_{34}}-u_{3}-v_{4}=23.48
\end{array}
$$

Since, all the reduced cost for the non-basic variable $\frac{\partial f}{\partial x_{N B_{i j}}} \geq 0$ at $\bar{x}^{1} \quad$ (KKT optimality point), it means the current solution is optimal. Therefore, from the above mathematical calculations using KKT optimality algorithm on a hypothetical data, it is observed that the optimal solution that give maximum achievable per unit profit are the supply of 50 units of $\mathrm{E}$ to $\mathrm{B}$ at a discount of $3 \%, 100$ units of $\mathrm{E}$ to $\mathrm{C}$ at a discount of $5 \%, 100$ units of $\mathrm{F}$ to $\mathrm{A}$ at a 
discount of $3 \%, 100$ units of $\mathrm{F}$ to $\mathrm{D}$ at a discount of $2 \%, 200$ units of $\mathrm{G}$ to $\mathrm{B}$ at a discount of $4 \%$.

\section{Conclusion}

In this paper, we have demonstrated that how fractional transportation problem can be solved efficiently for maximizing the per unit profit. This paper aimed at solving fractional transportation problem with volume discount on quantity of goods shipped which is a nonlinear fractional transportation problem. We applied KKT optimality algorithm to solve the fractional transportation problem. Maximization of profit is realized with discounts on large volumes, which means the determination of the best transportation route that would lead to low transportation cost and the effective transportation of these goods. This method can be used in all cases where discount cost, road tax, damage during the transportation is given. We then conclude that given discount on the cost of shipment could lead to increased productivity of producers. It provides the decision maker an alternative view to finding out the positive extent while transporting a product and hence this technique can contribute significantly to the literature of fractional programming.

\section{Acknowledgements}

The authors would like to thank the anonymous referees for constructive comments on an earlier version of this paper.

\section{References}

1. Acharya D. Basu M.and Das A. (2013). Discounted generalized transportation problem. International Journal of Scientific and Research Publications, 3(7): 1-6.

2. Anholcer M. (2015). The non-linear generalized transportation problem with convex costs. Croatian Operational Research Review, 6(1): 225-239.

3. BitranG.R. and Novaes A.G. (1973). Linear programming with a fractional function. Operations Research,21(4): 22-29

4. CaoB. and Uebe G. (1995). Solving transportation problems with non-linear side constraints with tabu search. Computers and Operations Research, 22(6): 593603.

5. Charnes A. and Cooper W.W. (1954). The stepping stone method of explaining linear programming calculations in transportation problems. Management Science, 1(1): 49-69. 
6. Charnes A. and Cooper W.W.(1962). Programs with linear fractional functions. Naval Research Logistics Quarterly,9: 181-196.

7. Cosares S. and Hochbaum D.S.(1994). Strongly polynomial algorithms for the quadratic transportation problem with a fixed number of sources. Mathematics of Operations Research, 19(1): 94-111.

8. Dangalchev C.A. (1996). Partially-linear transportation problems. European Journal of Operational Research, 91: 623-633.

9. Dantzig G.B.(1951). Application of the simplex method to a transportation problem. Activity Analysis of Production and Allocation, 13: 359-373.

10. Dhingra R. Jain M. Singh, P. and Saxena P.K. (2012). An algorithm to solve multiobjective fractional bottleneck transportation problem with restrictions. Asian Journal of Current Engineering and Mathematics, 1: 271-278.

11. Ekezie D. D. and Opara J. (2013). The application of transportation algorithm with volume discount on distribution cost (A case study of Port Harcourt Flour Mills Company Ltd.). Journal of Emerging Trends in Engineering and Applied Sciences, 4(2): 258-272.

12. Gupta S. Raina A.A. Ali I. and Ahmed A (2018). SAI method for solving job shop sequencing problem under certain and uncertain environment. Sri Lankan Journal of Applied Statistics. Accepted (In Press)

13. Haruna I. Ahmed M. and Emmanuel A. (2012). The network transportation problem with volume discount on shipping cost.International Journal of Science and Research, 3(12): 1840-1843.

14. Hitchcock F. L. (1941). The distribution of a product from several sources to Numerous Localities. Journal of Mathematical Physics, 20: 224-230.

15. Ilich N. and Simonovic S.P. (2001). An evolution program for non-linear transportation problems. Journal of Heuristics, 7: 145-168.

16. Jain M. and Saksena P.K. (2012). Time minimizing transportation problem with fractional bottleneck objective function. Yugoslav Journal of Operations Research,22(1): 115-129.

17. Jo J.B. Li Y. and Gen M. (2007). Non-linear fixed charge transportation problem by spanning tree-based genetic algorithm. Computers \& Industrial Engineering, 53: 290-298.

18. KlansekU. and Psunder M. (2010). Solving the non-linear transportation problem by global optimization. Transport, 25(3): 314-324.

19. Liu S.T. (2016). Fractional transportation problem with fuzzy parameters. Soft Computing, 20(9): 3629-3636.

20. Mubashiru A.S.S. (2014). Transportation with volume discount: a case study of a logistic operator in Ghana. Journal of Transport Literature, 8(2): 7-37. 
21. Nuran G. Ybrahim E. Fatih T. Copkun G. and Mustafa S. (2012). A solution proposal to the Interval Fractional Transportation Problem. International Journal of Applied Mathematics \& Information Sciences, 6(3): 567-571.

22. Sadia S. Gupta N. and Ali Q. M. (2018). Multi-objective fractional transportation problem in fuzzy environment. International Journal of Mathematical Archieve, 8(12):197-209.

23. Safi M. and Ghasemi S.M. (2017). Uncertainty in linear transportation problem. Int. J. Nonlinear Anal. Appl., 8(1): 81-93.

24. Shetty C.M. (1959). A solution to the transportation problem with non-linear costs. Operation Research, 7(5):571-580.

25. Singh P. (2015). Multiple-objective fractional costs transportation problem with bottleneck time and impurities. Journal of Information and Optimization Sciences, 36(5): 421-449.

26. Swarup K. (1966). Transportation technique in linear fractional functional programming. Journal of Royal Naval Scientific Service, 21(5): 256-260.

27. Tuy H. GhannadanS. MigdalasA. and Varbrand P. (1993). Strongly polynomial algorithms for a production-transportation problem with concave production cost. Optimization, 27: 205-227.

28. Tuy H. Ghannadan S. Migdalas A. and Varbrand P. (1996). A strongly polynomial algorithm for a concave production-transportation problem with a fixed number of non-linear variables. Mathematical Programming, 72(3): 229-258. 